\title{
A Survey of Current Work in Medical Text Mining---Data Source Perspective
}

\author{
Li Yanhong, Song Anmeng, Wang Jingling
}

School of Information Management and Engineering, Shanghai University of Finance and Economics, Shanghai, China

\author{
Email address: \\ 840931786@qq.com (Wang Jingling)
}

To cite this article:

Li Yanhong, Song Anmeng, Wang Jingling. A Survey of Current Work in Medical Text Mining---Data Source Perspective. International Journal of Biomedical Science and Engineering. Vol. 5, No. 3, 2017, pp. 29-34. doi: 10.11648/j.ijbse.20170503.13

Received: July 30, 2017; Accepted: August 31, 2017; Published: September 14, 2017

\begin{abstract}
This paper discusses the application of text mining in medical field at home and abroad based on overview and analysis of current literature data. Foreign researchers have specific text mining tools, and they use it in the search engine data and electronic medical record. In addition, it is also used to predict side effects between drugs. In China, text mining based on medical literature data occupies a large part. On the one hand, they can monitor the self disclosure of health information. On the other hand, they explore whether online information can help individuals get out of the disease. With the development of information technology, text mining will become more and more widely applied in the medical field in the future.
\end{abstract}

Keywords: Medical Field, Text Mining, Data Source

\section{Introduction}

With the rapid development of medical and health information construction process, the type and size of medical data is growing at an unprecedented pace, so that they cannot be easily collected, managed and integrated by current mainstream software tools within a reasonable time, therefore the hospital cannot take advantages of the advanced system to help make more positive decisions which could assist those who suffer from disease. There are four common sources of content-rich medical data. Firstly, the data pharmaceutical companies and life science. The data generated by drug discovery is quite dense, even for small and mid-sized enterprises, the data reaches the scale of TB. In the field of life sciences, with the gradual increase in computing capacity and gene sequence testing capabilities, John Wiley argues that there will be more people who have genetic maps, and for a single genomic sequence file, the size is about 750MB. Secondly, clinical medicine. Lumping the data from clinical and laboratory together, the medical institutions are faced with a fast-paced growth in medical data. For example, a CT image contains about $150 \mathrm{MB}$ of data and a standard pathological picture is close to 5GB. Thirdly, information about expenses and medical insurance generated during the medical treatment of patients is also another source of medical data. Fourthly, healthcare social media.
With the rapid development of mobile devices and mobile Internet and the popularity of social media and physiological portable devices, the data stored in each health communities is immeasurable.

Text mining, which is also called text-mining technique, is the discovery by computer of new and previously unknown information, such as interesting patterns and relationships in textual data, by automatically extracting information from different written texts. Text mining is bound up with data mining, an important research area focused on the extraction of useful information from data records. However, text mining has proven to be more difficult than data mining, as the data source consists of numerous unstructured texts rather than structured data in well-defined databases.

The goal of this article is to summarize the application of text-mining technique in medical domain. This article has two separate perspectives, the states of foreign and domestic fields respectively. In terms of the foreign research, they not only focus their attentions on how to mine data from the website and social media, but also on developing professional software to help they detect specific disease in journals or professional databases. Therefore, this article will make a brief introduce in this two sections. With regard to the domestic research, the primary attempts are the medication regularity of a particular disease through traditional Chinese medicine (TCM), in the same time, some western medicine's 
drugregularities. Therefore, it is worthy for subsequent researchers to concisely understand the current states of development in medical text mining and their drawbacks as well as the future directions.

\section{Research Review}

\subsection{Foreign Research}

Foreign researchers may be much more ahead in the development of medical text mining than domestic community. Their works are more extensive, the data are more wide-ranged, from journals and databases to website and social media to explorer and electronic medical record. And their objectives are much more different with each other with their own novel viewpoint.

\subsubsection{Text Mining in Journals and Databases}

Luis Tari, Saadat Anwar, Shanshan Liang \& James Cail etc (2010) discover drug-drug interactions (DDI) through an innovative method which integrates text-mining tool [1]. Using this approach, they can not only detect the explicit interactions but also the potential interactions that could be derived through the use of logic representation of the domain knowledge and automated reasoning. Hodong Lee, Gwan-Su Yi \& Jong (2008) focus their attention primarily on aubiquitin-protein ligase called E3 [2]. They develop E3Miner which is a web-based text-mining tool that extracts and organizes comprehensive and complete knowledge about E3s from the abstracts of journal articles and the relevant databases, providing easily access to users with a good grasp of E3s and their related information. The tool analyzes text sentences to identify protein names for E3s and also retrieves E3 data about protein functions, other E3-interactingpartners and E3-related human diseases from the protein databases, which could provide us more useful information.

\subsubsection{Text mining in Website and Social Media}

Courtney, Diane, Armin \& Karan (2010) argue that the web and social media (WSM) is just like a pool of data, which offers us important disease surveillance resource [3]. In traditional method, a patient with influenza-like illness (ILI) will be checked by a physician, so it takes time to conduct a diagnostic test or take blood samples which could provide a certain diagnosis. Thus it is difficult to identify the trend of flu since many cases of influenza remain puzzled about what disease they have. On the contrary, the text-mining research can use mining information to identify the flu trend. Meanwhile, they have proved that there exists a significant correlation between the detected increase in ILI and the patients reporting of Centers for Disease Control and Prevention (CDC) during a 24-week period from 5 October 2008 to 21 March 2009. Moreover, graph-based algorithms are not only available for ranking communities that discuss and disseminate information of influenza, but also can facilitate bio-event detection by searching for anomalies in WSM. According to the fact that twitter users often report a combination of symptoms, rather than a suspected or final diagnosis, using naive and everyday language to describe their diseases, Gesualdo, Stilo \& Agricola (2013) found that twitter has the potential to be a rich-content and cost-effective source of data for syndromic surveillance [4]. To prove their assumptions, they developed a minimally trained algorithm that exploits the abundance of health-related web pages to identify all jargon expressions related to a specific technical term. Then the definition of an influenza case had been translated into a Boolean query, each symptom being described by a technical term and all related jargon expressions, as identified by the algorithm above. After monitored all tweets that reported a combination of symptoms and put them into the model above, they found a high correlation coefficient between the trend of influenza-positive tweets and real-life ILI trends registered by US traditional surveillance systems. Nikfarjam A, Sarker A\&O'Connor K etc. (2015) attempt to develop a machine learning-based approach to extract mentions of adverse drug reactions (ADRs) from highly informal and descriptive text in social media [5]. They introduce a machine learning-based concept extraction system, namely ADR Mine, to model words' semantic similarities by cluster approach. Using deep learning technique, they generate pretrained word representation vectors with unlabeled user posts without supervision. It turns out that ADR Mine outperforms several standard systems in ADR extraction, reaching an F-measure of 0.82. It supposes that word cluster features remarkably improve extraction performance and weaken he demand for large, annotated training data sets. Apart from disease surveillance and extraction of ADRs, it turns out that the web and social media (WSM) is also full of abuse-related information which could be used to monitor prescription medication abuse by Abeed Sarker, Karen O'Connor \& Rachel Ginn etc. (2016) [6]. They take three commonly used abused medications (Adderall, Oxycodone and quetiapine) for testing by collecting Twitter user posts after having manually annotated 6400 tweets mentioning these three medications. Through training, they design an automatic supervised classification technique to determine whether the content contains signals of medication abuse or not. To testify the utility of automatic classification approach, they also analyze abuse pattern over time using this classification data, achieving $82 \%$ accuracy overall.

\subsubsection{Text mining in Search Engine Query}

Ginsberg, Mohebbi, Matthew \& Patel etc. (2009) explored another aspect of text-mining area by detecting influenza epidemics with search engine query data [7]. They not only desire to discover seasonal influenza epidemics, which cause tens of millions of respiratory illnesses and deaths worldwide each year, but more importantly the new influenza virus against which no previous immunity exists. They believed that early detection and surveillance of health-seeking behavior in the form of queries to online search engines may provide a relief when followed by a rapid response. Consequently, they supposed a method of analyzing large numbers of Google search queries to track influenza-like 
illness within specific population based on the theory that the relative frequency of certain queries is highly correlated with the percentage of patients who have influenza-like symptoms. The supposed approach could be able to detect influenza epidemics in areas of United States with a large population of web search users by their search queries. Philip, Yiling, David \& Forrest (2008) started from the intuitive assumption that the frequency of Internet searches may provide information regarding infectious disease activity [8]. Thus they conducted experiment to testify the relationship between search queries contained influenza-related search terms in the Yahoo! search engine for influenza from March 2004 through May 2008 and actual influenza occurrence in the United States. By using searches with 1-10-week lead times as explanatory variables, linear models was built to predict the percentage of cultures positive for influenza and deaths attributable to pneumonia and influenza in the United States. The result showed that their models predicted an increase in cultures positive for influenza 1-3 weeks in advance of when they occurred, as well as an increase in mortality attributable to pneumonia and influenza up to 5 weeks in advance. Search-term surveillance may provide an additional tool for disease surveillance. Hulth, Rydevik \& Linde (2009) calculated the occurrence of various queries related to influenza from search logs submitted to a Swedish medical web site for two influenza seasons [9]. These figures were subsequently used to generate two models, one to estimate the number of laboratory verified influenza cases and one to estimate the proportion of patients with influenza-like illness reported by selected General Practitioners in Sweden. Particularly, they applied an approach designed for highly correlated data, partial least squares regression. The result demonstrated that certain web queries on influenza follow the same pattern as that obtained by the two other surveillance systems for influenza epidemics, and that they have equal power for the estimation of the influenza burden in society. However, web queries are an accurate, cheap and labor extensive alterative for syndromic surveillance. Samaras, Garcia, Elena \& Sicilia etc. (2012) found that many recent research has paid attention to the potential of Web queries as a source for syndromic surveillance and the ability for estimation and prediction of the development of a syndromic disease, such as using linear model to detect influenza [10]. But they went further to build a new statistical method to prove the relationship between related search engine queries with scarlet fever cases in the UK and the real data obtained from the official agencies. After optimizing, another alternative statistical method based on gamma distributions was built and could obtain better results in all cases, which means the model has the ability to predict the peak and the spread of the distributions that beyond the capability of other models, especially in those with a smaller correlation factor.

\subsubsection{Text Mining in Electronic Medical Records}

Luther, Mc Cart, Berndt \& Hahm etc. (2015) determined whether statistical text mining (STM) can identify fall-related injuries in electronic health record (HER) documents and the impact on STM models of training on documents from a single or multiple facilities [11]. STM models based on training data from a single facility resulted in accuracy of $87.5 \%$ and $87.1 \%$, F-measure of $87.0 \%$ and $90.9 \%$, sentivity of $92.1 \%$ and $94.1 \%$, and specificity of $83.6 \%$ and $77.8 \%$ at the visit and patient levels, respectively. Results from training data from multiple facilities were almost identical. STM has the potential to improve identification of fall-related injuries in the VHA, providing a model for wider application in the evolving national HER system. Hammond, Ben, Laundary \& Samore etc. (2015) applied text-mining methodology to study clinical questions in large populations [12]. To assess the feasibility of text mining, investigation of the relationship between exposure to adverse childhood experiences (ACEs) and recorded diagnoses was conducted among all VA-treated Gulf war veterans, utilizing all progress notes recorded from 2000-2011. Text mining to detect ACE exposure in a large population was feasible. Analysis of the relationship between ACE score and adult health conditions yielded patterns of association consistent with prior research. Duggal, Shukla \& Chandra etc. (2016) classified the patients into two different risk groups of readmission within 30 days of discharge based on patients' characteristics using 2-year clinical and administrative data [13]. It proposed architecture of this prediction model and identified various risk factors using text mining techniques. Also, groups of consistently occurring factors that inference readmission rates were revealed by associative rule mining, It then evaluated the classification accuracy using five different data mining classifies and conducted cost analysis, Out of total 9381 records, 1211 encounters were found as readmissions. This study concludes that the model could be incorporated in healthcare institutions to witness its effectiveness. Cost analysis shows huge savings which is significant for any healthcare system especially in developing counties like India. Casillas, Perez \& Oronoz etc. (2016) extracted adverse drug teaction events from electronic health records in Spanish [14]. They present a hybrid system utilizing a self-developed morpho-syntactic and semantic analyzer for medical texts in Spanish. It performs named entity recognition of drugs and diseases and adverse drug reaction event extraction. The event extraction stage operates using rule-based and machine learning techniques. One of the contributions of the machine learning based system is its ability to deal with both intra-sentence and inter-sentence events in a highly skewed classification environment. Moreover, the knowledge-based and the inferred model are complementary in terms of precision and recall. While the former provides high precision and low recall, the latter is the other way around. As a result, an appropriate hybrid approach seems to be able to benefit from both approaches and also improve them. This is the underlying motivation for selecting the hybrid approach. In addition, this is the first system dealing with real electronic health records in Spanish. Ford, Carroll \& Smith etc.(2016) extracted information from the text of electronic medical records to improve case detection: a systematic review [15]. Electronic medical records (EMRs) are revolutionizing 
health-related research. One key issue for study quality is the accurate identification of patients with the condition of interest. Information in EMRs can be entered as structured codes or unstructured free text. The majority of research studies have used only coded parts of EMRs for case-detection, which may bias findings, miss cases, and reduce study quality. This review examines whether incorporating information from text into case-detection algorithms can improve research quality. Text in EMRs is accessible, especially with open source information extraction algorithms, and significantly improves case detection when combined with codes. More harmonization of reporting within EMR studies is needed, particularly standardized reporting of algorithm accuracy metrics like positive predictive value and sensitivity.

\subsection{Domestic Research}

Under the circumstance of data explosion, it is necessary and important to explore the large-scale of medical data by text-mining technique. In domestic research, our scientists pay their attention primarily on the regularity of traditional Chinese medicine towards a particular disease, occasionally they may compare the different treatment of traditional Chinese medicine between certain diseases. Few researchers follow foreign researchers' pace, how to utilize the information in web and social media to help government to monitor disease and individual to relief is their focus.

\subsubsection{Text Mining Technique Towards a Particular Disease}

The relationship among symptom, pattern and medication regularities of hypertension have been investigated by $\mathrm{He}$ Dan, Jiang Miao, Zheng Chi etc. (2014) through applying text-mining technique [16]. Though analysis of the data in relevant literature, they arrived at the conclusion subjected to data slicing algorithm. The results are that the most common symptoms of hypertension are headache (2650), dizziness (1734) and the most injured organ are liver and kidney and the most useful herb are Gastrodiae Rhizoma and Uncaria Ramulus Cum Uncis in TCM. Guo Hongtao, Zheng Guang, Zhao Jing etc. (2011) used text-mining technique to download raw data from the Chinese Bio Medical Literature Database (CBM) with the keyword H1N1 [17]. After preprocessing, the data was mined for traditional Chinese therapeutic regularities in treatment of influenza H1N1 based on the discrete derivative algorithm. It is shown that therapy of influenza H1N1 concentrates on dispelling heat and resolving toxicity in TCM, that the widely used TCM medicines are Lian-Hua-Qing-Wen Capsule, Tan-Re-Qing Injection, Qing-Kai-Ling Injection, that the syndrome of influenza H1N1 can be defined by toxicity, heat and phlegm when using TCM pattern. The medication regularities of chronic gastritis have been explored by $\mathrm{Li} \mathrm{Li}$, Zhou Qi, Zheng Guang etc. (2011) based on text-mining technique [18]. The data are collected from the rich-content literature in CBM, they through preprocessing, the raw, unstructured XML type data are transferred into structured data into the database. To show the conclusion, different kinds of graphs are introduced by Cytoscape software, visualizing the result of their research. It is suggested that the most frequently used Chinese patent medicine are Sini San as well as Lizhong Wan.

\subsubsection{Text Mining Technique Towards Disease Comparison}

Guo Hongtao, Zheng Guang, Zhang Chi etc. (2010) started from the famous saying in TCM, treating different with the same therapy, since some diseases have the same principle in TCM sense, such as the relationship between Rheumatoid Arthritis (AR) and Diabetes Mellitus (DM) [19]. In the paper, a text-mining algorithm based on the word frequency has been proposed to mine the data from literatures in CBM with the keywords AR and DM. It is suggested that the Chinese herb Huang Qi, Dang Gui are the commonly core medicine for both AR and DM, as their functions are blood-activating and stasis-resolving. The previous assumption proves to be a solid scientific foundation. Another aspect of hypertension, the comparison of regularities of Chinese herbs, Chinese patent drug and western medicine in treatment, is being analyzed by Wang Liying, Zheng Guang \& Guo Hongtao etc. (2013) using the text-mining technique [20]. Literatures about hypertension from CBM have been extracted, and then subjected to data process. They concluded that gastrodia elata and uncaria rhynchophylla were most commonly used Chinese herbs, injection of red sage root and zhenju jiangya tablet were most pervasive Chinese patent drug, captopril and nifedipine were most popular western medicine in the treatment of hypertension. What's more, they went further with the best combination of Chinese patent drug and western medicine, including captopril and zhenju Jiangya tablet, nifedipine and zhenju jiangya tablet.

\subsubsection{Text Mining in Website and Social Media}

$\mathrm{Ku}$, Chiu \& Zhang etc. (2014) explored self-disclosing health information to support public health surveillance and healthcare using data from two major HIV/AIDS forums (Yahoo! Knowledge and Taiwan AIDS Foundation forum) in Taiwan [21]. The framework they built includes an incremental web crawler program to acquire data and the Stanford Word Segmenter to parse sentence based on simplified Chinese. Additionally, several feature sets (bag-of-words, word/POS, bag-of-words+word/POS, selected bag-of-words+word/POS, and DSF) were generated from the collected postings by different text representation approaches, coupled with SVM and NB algorithms to train the classifier for identifying unusual messages. Their result has shown the effectiveness of applying text mining techniques on HIV/AIDS-related self-disclosing health information on web forums as well as the promising applicability to healthcare practice, such as other self-disclosing health information issues (e.g. suicide, weight loss). Lu Yan and Yong Tan (2014) create a new perspective, they investigate whether social support exchanged in an online healthcare community benefits patients' mental health [22]. Social support exchange means that patients can login in an online community where they can get the answer and write their puzzles about particular disease. In order to 
measure the social support, firstly they classified social support into three kind, informational support, emotional support and companionship. Secondly, they proposed a non-homogeneous Partially Observed Markov Decision Process (POMDP) model to examine possible health outcomes for the members. What they found was that patients benefit from learning for mothers and that their participation in the online community helps them to improve their health and to better engage in their disease self-management process. It was also shown that different forms of social support exchange have different influence on patients' health conditions, among which informational support is the most typical type and emotional support plays the most vital role in helping patients move to a healthier state. Finally, the proposed POMDP model can predict patients' health states with moderate accuracy and has the ability to replenish missing or unavailable statement of patients' health conditions.

\section{Conclusion}

In the era of big data, how to quickly access the information in medical resources, and how to use relevant information to enhance the medical experience of human beings is a realistic problem that needs to be solved in the future. Foreign researchers may be much more ahead in the development of medical text mining than domestic community. Their main work can be divided into four separate parts. Firstly, they develop specific tools or programs for their unique demand, ranging from the information of aubiquitin-protein ligase to drug-drug interactions. Secondly, the web and social media (WSM) has been regarded as another rich resource of medical data, many attempts have been taken to detect disease trends, find content containing adverse drug reactions and monitor prescription medication abuse. Thirdly, the application of text-mining tools in search engine query data is another area for disease detection and surveillance. Finally, the application of text-mining tools in electronic medical records is another method to predict the probability of some disease. Moreover researchers can find the interaction between drugs. In addition it was used in predicting side effects between drugs and improving a case detection.

In domestic community, literature-based researches account for the most part of all endeavors, such as the literature from database CBM. The difference between researchers is that some use abstracts of specific articles, but others think full-text is more comprehensive. Their theme concentrates on the regularity of traditional Chinese medicine no matter for single disease or disease comparison, including hypertension, H1N1, flu, chronic gastritis, diabetes mellitus and rheumatoid arthritis. Few researchers spare effort on the information pool in web and social media recently. On one hand, they can monitor self-disclosing health information for healthcare institutions, on the other hand, they explore whether the online information could help individual to relief from disease themselves.
In conclusion, the researchers focus on the basic methods and techniques of text mining in medical field and its application in medicine and related disease. However the research about text mining in disease misdiagnosis, clinical pathological study, and mental disease is relatively few. Researchers can use text mining in these aspects. Moreover with the algorithms of text mining are constantly improved. Researchers can find more value information in medical field. In the future text mining will become more and more widely applied in the medical field.

\section{Acknowledgements}

This work was supported partly by the National Science Foundation of China (No.7140106).

\section{References}

[1] Luis Tari, Saadat Anwar, Shanshan Liang \& James Cail etc. (2010) Discovering drug-drug interactions: A text-mining and reasoning approach based on properties of drug metabolism, bioinformatics Vol. 26, pages i547-i553.

[2] Hodong Lee, Gwan-Su Yi \& Jong (2008) E3Miner: a text mining tool for ubiquitin-proteinligases, Nucleic Acids Research, Vol. 36W416-W422.

[3] Courtney, Diane, Armin\& Karan (2010) Text and Structural Data Mining of Influenza Mentions in Web and Social Media Int. J. Environ. Res. Public Health, 7, 596-615.

[4] Gesualdo, Stilo \& Agricola (2013) Influenza-Like Illness Surveillance on Twitter through Automated Learning of Naïve Language, PLoS ONE., Vol. 8 Issue 12, p1-1.

[5] Nikfarjam A, Sarker A \& O'Connor K etc. (2015) Pharmacovigilance from social media: mining adverse drug reaction mentions using sequence labeling with word embedding cluster features, J Am Med Inform Assoc. 22(3): 671-81.

[6] Abeed Sarker, Karen O'Connor \& Rachel Ginn etc. (2016) Social Media Mining for Toxicovigilance: Automatic Monitoring of Prescription Medication Abuse from Twitter, Drug Saf. 39: 231-240.

[7] Ginsberg, Mohebbi, Matthew \& Patel etc. (2009) Detecting influenza epidemics using search engine query data, Nature 457, 1012-1014.

[8] Philip, Yiling, David \& Forrest (2008) Using Internet Searches for Influenza Surveillance, Healthcare Epidemilogy • CID 1443-1448.

[9] Hulth, Rydevik \& Linde (2009) Web Queries as a Source for Syndromic Surveillance, PLoS ONE., Vol. 4 Issue 2, p1-10.

[10] Samaras, Garcia, Elena \& Sicilia etc. (2012)Syndromic surveillance models using Web data: The case of scarlet fever in the UK, Informatics for Health \& Social Care. Vol. 37 Issue 2, p106-124.

[11] Luther, Mccart \& Berndt etc. (2015) Improving Identification of Fall-Related Injuries in Ambulatory Care Using Statistical Text Mining, American Journal of Public Health, 105(6): 1168-73. 
[12] Hammond, Benari \& Laundry etc.(2015) The Feasibility of Using Large-Scale Text Mining to Detect Adverse Childhood Experiences in a VA-Treated Population, Journal of Traumatic Stress, 28(6): 505.

[13] Duggal, Shukla \& Chandra etc. (2016) Predictive risk modeling for early hospital readmission of patients with diabetes in India, Internation Journal of Diabeted in Developing Countries, 1-10.

[14] Casillas, Perez \& Oronoz etc. (2016) Learning to extract adverse drug reaction events from electronic health records in Spanish, Expert Systems with Applications, 61: 235-245.

[15] Ford, Carroll \& Smith etc. (2016) Extracting information from the text of electronic medical records to improve case detection: A systematic review, J Am Med Inform Assoc, Vol. 5: 180.

[16] He Dan, Jiang Miao, Zheng Chi etc. (2014) Exploring Relationship Among Symptom, Pattern and Medication Regularity of Hypertension Based on Text Mining Technology, Chinese Journal of Experimental Traditional Medical Formulea, Vol. 20, No. 19, pages 214-216.

[17] Guo Hongtao, Zheng Guang, Zhao Jing etc. (2011) Traditional Chinese Medicine Therapeutic Characteristics in the Treatment of Influenza A Virus Subtype H1N1 Based on Data Mining, World Science and Technilogy/Modernization of Traditional
Chinese Medicine and Materia Medica, Vol. 13, No. 5, pages 772-776.

[18] Li Li, Zhou Qi, Zheng Guang etc. (2011) Based on Data Mining Techniques to Explore Medication Regularity of Chinese Patent Medicine and West Medicine Application for Chronic Gastritis, Chinese Journal of Experimental Traditional Medical Formulea, Vol. 17, No. 24, pages 228-231.

[19] Guo Hongtao, Zheng Guang, Zhang Chi etc. (2010) Exploring Commonly Existed Networks of Chinese Herbal Medicines Used in Rheumatoid Arthritis and Diabetes Mellitus through Data Mining, World Science and Technilogy/Modernization of Traditional Chinese Medicine and Materia Medica, Vol. 12, No. 5 , pages $818-822$.

[20] Wang Liying, Zheng Guang \& Guo Hongtao etc. (2013) Regularity of clinical medication of hypertension analyzed with text mining approach, Institute of Basic Research In Clinical Medicine, China Academy of Chinese Medical Sciences, Vol. 28, No. 1, pages 60-63.

[21] Ku, Chiu \& Zhang etc. (2014) Journal of the Association for Information Science and Technology, 65(5): 928-947.

[22] Lu Yanand Yong Tan (2014) Feeling Blue Go Online: An Empirical Study of Social Support Among Patients, Information Systems Research, Vol. 25, No. 4, pp. 690-709. 